\title{
Amplification and dampening of soil respiration by changes in temperature variability
}

\author{
C. A. Sierra ${ }^{1, *}$, M. E. Harmon ${ }^{1}$, E. Thomann ${ }^{2}$, S. S. Perakis ${ }^{3}$, and H. W. Loescher ${ }^{4}$ \\ ${ }^{1}$ Department of Forest Ecosystems and Society, Oregon State University, Corvallis, Oregon 97333, USA \\ ${ }^{2}$ Department of Mathematics, Oregon State University, Corvallis, Oregon 97333, USA \\ ${ }^{3}$ US Geological Survey, Forest and Rangeland Ecosystem Science Center, Corvallis, Oregon 97333, USA \\ ${ }^{4}$ National Ecological Observatory Network, Boulder, Colorado 80301, USA \\ *now at: Max-Planck-Institute for Biogeochemistry, 07745 Jena, Germany
}

Received: 2 December 2010 - Published in Biogeosciences Discuss.: 10 December 2010

Revised: 4 April 2011 - Accepted: 13 April 2011 - Published: 19 April 2011

\begin{abstract}
Accelerated release of carbon from soils is one of the most important feedbacks related to anthropogenically induced climate change. Studies addressing the mechanisms for soil carbon release through organic matter decomposition have focused on the effect of changes in the average temperature, with little attention to changes in temperature variability. Anthropogenic activities are likely to modify both the average state and the variability of the climatic system; therefore, the effects of future warming on decomposition should not only focus on trends in the average temperature, but also variability expressed as a change of the probability distribution of temperature. Using analytical and numerical analyses we tested common relationships between temperature and respiration and found that the variability of temperature plays an important role determining respiration rates of soil organic matter. Changes in temperature variability, without changes in the average temperature, can affect the amount of carbon released through respiration over the longterm. Furthermore, simultaneous changes in the average and variance of temperature can either amplify or dampen the release of carbon through soil respiration as climate regimes change. These effects depend on the degree of convexity of the relationship between temperature and respiration and the magnitude of the change in temperature variance. A potential consequence of this effect of variability would be higher respiration in regions where both the mean and variance of temperature are expected to increase, such as in some low latitude regions; and lower amounts of respiration where the average temperature is expected to increase and the variance to decrease, such as in northern high latitudes.
\end{abstract}

Correspondence to: C. A. Sierra (csierra@bgc-jena.mpg.de)

\section{Introduction}

One of the most important feedbacks of terrestrial ecosystems to climate change is the potential release of soil carbon as temperature increases, especially at high latitudes (Field et al., 2007). The amount of carbon stored in soils worldwide exceeds the amount of carbon in the atmosphere by a factor of two to three (Houghton, 2007), and there is concern that a large portion of this carbon will be released to the atmosphere as the global average temperature increases (Schimel et al., 1994; Kirschbaum, 1995; Trumbore, 1997; Davidson and Janssens, 2006). Predictions of this positive feedback have focused on the effects of increasing average temperatures with little attention to possible effects of changes in temperature variability. There is already evidence of important changes in temperature variability at the regional scale and climate models predict even larger changes for the next decades (Räisänen, 2002; Brönnimann et al., 2007; IPCC, 2007; Schar et al., 2004; Meehl et al., 2009); hence, the effects of future warming on decomposition should not only focus on trends in the average, but more broadly on changes in the probability distribution of temperature. The frequency of hot or cold days and extreme events over long periods of time can potentially determine the frequency of large respiration pulses and subsequently the total amount of $\mathrm{C}$ stored in an ecosystem.

Historically, the theory behind the temperature dependency of organic matter decomposition has been supported by the equations that relate chemical reaction rates with temperature. The van't Hoff and Arrhenius equations have been widely used to predict changes in decomposition rates and respiration rates with changes in temperature. An increase in the respiration rates as temperature increases is expected under this theory, and it can be shown that these functions are convex within the interval of temperatures where most

Published by Copernicus Publications on behalf of the European Geosciences Union. 
Table 1. Empirical equations commonly used to describe the relationship between temperature $T$ and soil respiration $R$, with their second derivatives and range of convexity. $a, b$, and $c$ represent empirical coefficients. Equations extracted from Luo and Zhou (2006) and Del Grosso et al. (2005).

\begin{tabular}{|c|c|c|c|}
\hline Equation & Second derivative & Range of convexity on $T$ & Comments \\
\hline$R=a e^{b T}$ & $a b^{2} e^{b T}$ & $\begin{array}{l}-\infty<T<\infty, \text { if } a>0 \text { and } \\
b \neq 0\end{array}$ & $\begin{array}{l}\text { Van't Hoff's exponential function. } a \text { and } b \text { are } \\
\text { empiricial coefficients. }\end{array}$ \\
\hline$R=R_{0} Q_{10}^{\frac{T-T_{0}}{10}}$ & $R_{0}\left(\frac{\ln Q_{10}}{10}\right)^{2} Q_{10}^{\frac{T-T_{0}}{10}}$ & $\begin{array}{l}-\infty<T<\infty, \text { if } R_{0}>0 \\
\text { and } Q_{10} \neq 0\end{array}$ & $\begin{array}{l}\text { Modified van't Hoff. } R_{0}: \text { respiration rate at } \\
\text { temperature } T_{0} . Q_{10}: \text { relative increase by a } \\
10^{\circ} \mathrm{C} \text { change in temperature. }\end{array}$ \\
\hline$R=a e^{\left(-E_{\mathrm{a}} / \Re T\right)}$ & $a e^{\left(-E_{\mathrm{a}} / \Re T\right)}\left[\left(\frac{E_{\mathrm{a}}}{\Re T^{2}}\right)^{2}-\frac{2 E_{\mathrm{a}}}{\Re T^{3}}\right]$ & $\begin{array}{l}0<T<E_{\mathrm{a}} /(2 \Re), \text { if } E_{\mathrm{a}} \gg \\
\Re T\end{array}$ & $\begin{array}{l}\text { Arrhenius equation. } E_{\mathrm{a}} \text { : activation energy, } \Re \text { : } \\
\text { universal gas constant. } T \text { in Kelvin. }\end{array}$ \\
\hline$R=R_{10} e^{\left(\frac{E_{\mathrm{a}}}{283.15 \%}\right)\left(1-\frac{283.15}{T}\right)}$ & $R_{10} e^{\left(\frac{E_{\mathrm{a}}}{283.15 \Re}\right)\left(1-\frac{283.15}{T}\right)}\left[\left(\frac{E_{\mathrm{a}}}{\Re T^{2}}\right)^{2}-\frac{2 E_{\mathrm{a}}}{\Re T^{3}}\right]$ & $\begin{array}{l}0<T<E_{\mathrm{a}} /(2 \Re), \text { if } R_{10}> \\
0\end{array}$ & $\begin{array}{l}\text { Modified Arrhenius or Lloyd and Tay- } \\
\text { lor }(1994) \text { equation. } R_{10} \text { : respiration at } \\
10^{\circ} \mathrm{C} \text {. }\end{array}$ \\
\hline$R=a e^{b T+c T^{2}}$ & $a e^{b T+c T^{2}}\left[2 c+(b+2 c T)^{2}\right]$ & $\begin{array}{l}-\infty<T<\infty, \text { if } a>0, c> \\
0\end{array}$ & Second order exponential function. \\
\hline$R=a(T+10)^{b}$ & $a\left(b^{2}-b\right)(T+10)^{b-2}$ & $\begin{array}{l}-\infty<T<\infty, \text { if } a>0 \text { and } \\
b \neq 1\end{array}$ & Power function of Kucera and Kirkham (1971). \\
\hline$R=a+\frac{b \arctan (c \pi(T-15.7))}{\pi}$ & $\frac{-2 \pi c(\pi c(T-15.7))}{\left(1+(c \pi(T-15.7))^{2}\right)^{2}}$ & $\begin{array}{l}-\infty<T<15.7 . T=15.7 \\
\text { is an inflection point. For } \\
T>15.7, R \text { is concave. }\end{array}$ & $\begin{array}{l}\text { DAYCENT and CENTURY approach assum- } \\
\text { ing constant soil water content, Del Grosso } \\
\text { et al. (2005). }\end{array}$ \\
\hline
\end{tabular}

biological processes take place (Table 1). Convexity is also a property of other empirical equations used to represent the relationship between respiration and temperature, such as the modified van't Hoff equation and the equation developed by Lloyd and Taylor (1994).

If the relationship between temperature and respiration is convex for a given ecological or biological system, we can expect the predictions of Jensen's inequality (Jensen, 1906) to apply. According to Jensen's inequality, if temperature $T$ is a random variable, and $f(T)$ a convex function on an interval, then

$E[f(T)] \geq f(E[T])$,

where $E$ is the expected value operator. Evaluating the function with the mean of the random variable will produce a lower amount than taking the mean of the function's evaluations at each value of the random variable.

The implications of this inequality for temperaturerespiration studies are: (1) estimates of respiration from temperature data gathered at large spatial and temporal scales include bias or aggregation error (Rastetter et al., 1992; Kicklighter et al., 1994). (2) Modeling studies using average temperature as a driving variable will obtain lower values of respiration than if they were using temperature from weather records; (e.g., Notaro, 2008; Sierra et al., 2009; Medvigy et al., 2010). (3) Long-term incubation experiments at constant temperatures will result in lower respiration rates than experiments in which temperature is allowed to vary but without changing the average value.
Previous work on this subject has been focused on producing unbiased estimates of annual or large-scale respiration using temperature data that omits diurnal or seasonal fluctuations (Ågren and Axelsson, 1980; Kicklighter et al., 1994). In the context of global climate change a different question emerges. Would soils currently experiencing a given degree of temperature variability increase their respiration rates under a new climatic regime with different temperature variance? This question goes beyond of what can be predicted by Jensen's inequality alone because it implies changes in temperature variance that are originally not included in the inequality.

The effect of variability on respiration may depend on the magnitude and direction of the change in variance as well as the change in the average climate. Changes in temperature variability are associated with either an increase or decrease in the frequency of temperature extremes. Intuitively, we expect that this change in temperature variance would result in different rates of carbon release over the long-term depending on the convexity of the relationship between temperature and respiration. Furthermore, we expect that simultaneous changes in the mean and variance of temperature can potentially amplify or dampen the effect of changes in mean temperature alone. However, we lack a clear analytical framework to test these ideas and apply them in the context of global climate change and its effects on soil respiration.

In this study, we explored the effects of changes in temperature regimes, i.e., simultaneous changes in the mean and variance of temperature, on the potential amount of carbon release from ecosystems through soil respiration. This 
was accomplished by performing a mathematical analysis of the functions commonly used to predict soil respiration from temperature data. Since the consequences of changes in temperature variance are not easy to derive from Jensen's inequality alone, we first conducted an analytical analysis to find an explicit mathematical expression that summarizes these consequences. This was achieved by first exploring changes in temperature ranges in a geometrical context and subsequently more explicitly as changes in the variance of a random variable in a probabilistic context. To test these ideas with a broader scope, numerical simulations were performed with two different respiration functions with stochastic representations of realistic temperature time-series. In the latter analysis, three contrasting ecosystems were used for comparison purposes, an arctic tussock tundra, a temperate rain forest, and a tropical rain forest.

\section{Methods}

\subsection{Sites and datasets}

For the numerical analysis we used soil temperature data from three contrasting ecosystems, an arctic tussock tundra, a temperate rain forest, and a tropical rain forest.

Soil temperature data at $20 \mathrm{~cm}$ depth from the Toolik lake Long-Term Ecological Research (LTER) site in Alaska $\left(68^{\circ} 38^{\prime} \mathrm{N}, 149^{\circ} 43^{\prime} \mathrm{W}\right.$, elevation $760 \mathrm{~m}$ a.s.l.) were obtained from the site's webpage. The dataset contains daily temperature records from 1 June 1998 to 31 December 2006, with a grand mean of $-2.5^{\circ} \mathrm{C}$.

From the H. J. Andrews LTER site in Oregon we used soil temperature data measured at the PRIMET meteorological station $\left(44^{\circ} 12^{\prime} \mathrm{N}, 122^{\circ} 15^{\prime} \mathrm{W}\right.$, elevation $430 \mathrm{~m}$ a.s.1.). The record contains daily soil temperature values measured at $10 \mathrm{~cm}$ depth, starting on 26 December 1994 until 24 January 2007. The average temperature for this period was $11.8^{\circ} \mathrm{C}$. Detailed information about the soil temperature record can be found at the H. J. Andrews' website.

We collected high-frequency soil temperatures from La Selva Biological station in Costa Rica $\left(10^{\circ} 26^{\prime} \mathrm{N}, 83^{\circ} 59^{\prime} \mathrm{W}\right)$ to use in this analysis. Three platinum resistance thermometers were used to collect information at $5 \mathrm{~cm}$ depth with $2 \mathrm{~s}$ execution. Data were averaged to obtain 30 min time series that were further aggregated to obtain daily averages. The record extends from 1 January 1998 to 31 December 2000, and a global average of $23.7^{\circ} \mathrm{C}$.

\subsection{Data processing and modeling experiments}

The time series from the three sites were filtered with a nineday moving average to obtain an estimate of short-term vari- ability. In particular, if $T_{t}$ represents the daily temperature observations, then

$\tau_{t}=\frac{1}{9} \sum_{j=-4}^{4} T_{t-j}$,

where $\tau_{t}$ is a low-pass filter that captures the seasonal trend. An estimate of the short-term variability is then given by the residuals $\hat{\epsilon}_{t}=T_{t}-\tau_{t}$, with $E\left(\hat{\epsilon}_{t}\right) \approx 0$ and variance $\sigma_{\epsilon}^{2}>0$. The average annual trend can then be defined as $\overline{\tau_{d}}=\frac{1}{N} \sum_{n=1}^{N} \tau_{d, n}$, where $d=t-365(n-1)$ is the Julian day, $n$ is a year counter, and $N$ the total number of years in the time series.

We used $\overline{\tau_{d}}$ and $\sigma_{\epsilon}^{2}$ to simulate a reference climatic regime for the three sites, as well as departures to new climatic regimes. These changes in climatic regimes were performed within the framework provided by Mean versus Standard deviation Change (MSC) diagrams (Sardeshmukh et al., 2000; Scherrer et al., 2007). Within this framework it is possible to represent independent and simultaneous changes in the mean and variance of temperature time series (Fig. 1). For the purpose of this analysis we defined time series of reference climatic regimes as

$\tilde{T}_{t}=\bar{\tau}_{d}+\epsilon_{t}$

where $\tilde{T}_{t}$ is a simulated time series lengthened to a decade, i.e., $t=\{1, \ldots, 3650\}$. The term $\epsilon_{t}$ is a series of simulated Gaussian white noise, obtained as $\epsilon_{t} \sim \operatorname{iid} N\left(0, \sigma_{\epsilon}^{2}\right)$. Notice that the values of $\overline{\tau_{d}}$ are recycled 10 times until the end of the simulation.

New climatic regimes were simulated as a change in the mean and variance of the reference climatic regime by modifying the $\epsilon_{t}$ term in Eq. (3). A combination of values of mean $m$ and standard deviation $s$ were used to simulate time series with $\epsilon_{t} \sim \operatorname{iid} N\left(m, s^{2}\right)$, where $m=\{0.1,0.2, \ldots, 2\}$ and $s=\{0.1,0.2, \ldots, 3\}$. These values of $s$ were chosen arbitrarily, with the only requirement that the variance $\sigma_{\epsilon}^{2}$ in the three datasets were inside the range of the values in $s^{2}$. The values of $m$ and $s$ were chosen such that their combination can fill a wide domain of possibilities in the MSC plots. In other words, these combinations produce 600 different climatic regimes that are possible as the regional climate changes at each site. Notice that values of $m$ only reproduce warming from the starting conditions of the reference regime.

\subsection{Respiration models}

The simulated temperature regimes were used to run common empirical functions that relate temperature and respiration in terrestrial ecosystems. The modified van't Hoff model (Table 1) was used for the three ecosystems with an arbitrary value of the reference respiration $R_{0}=1$ at $T_{0}=10$, that is

$R_{t}=Q_{10}^{\frac{\tilde{T}_{t}-10}{10}}$. 
a
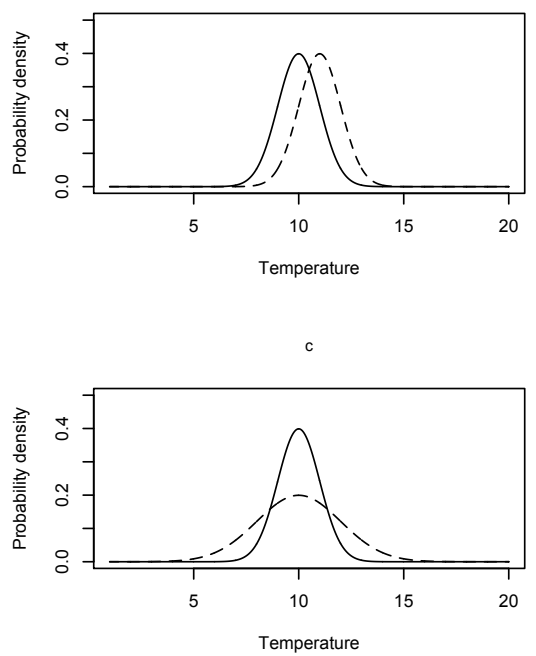

e

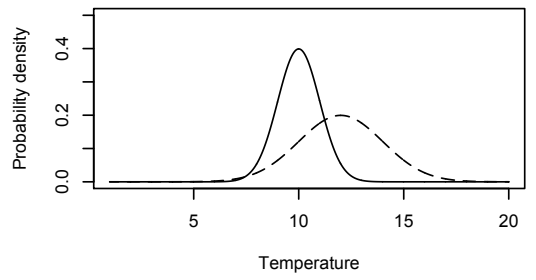

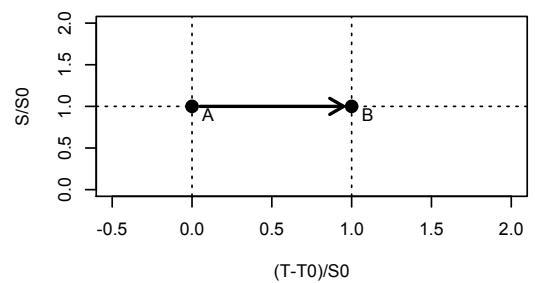
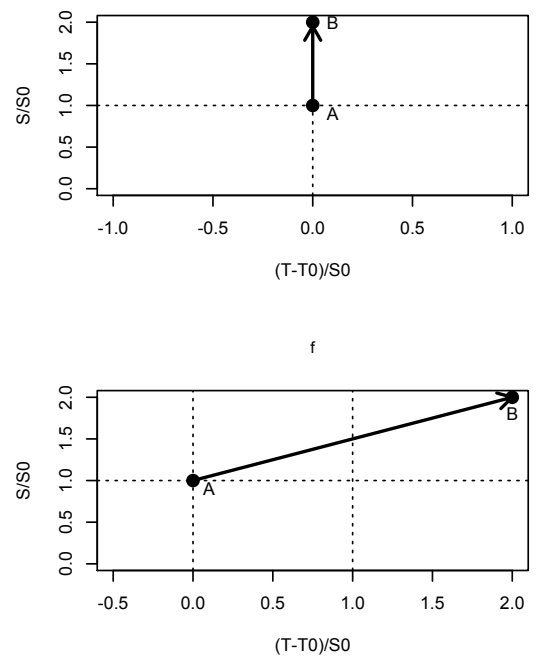

Fig. 1. Graphical representation of changes in the mean and standard deviation on a Gaussian climate distribution A with mean $T 0$ and standard deviation $S 0$ resulting in distribution B with mean $T$ and standard deviation $S$. (a) Probability density functions (pdfs) where the mean from distribution A (continuous) changes to distribution B (dashed), (b) distributions A and B from (a) in a standardized MSC plot showing standardized changes in the mean $(T-T 0) / S 0$ against standardized standard deviation $S / S 0$, (c) pdfs where the standard deviation from distribution A changes to distribution B. (d) standardized MSC plot of (c), (e) pdfs where both the mean and the standard deviation from A change to B, (f) standardized MSC plot of (e).

Since we are only interested in observing emergent patterns after using different climatic regimes we did not use site specific parameters in this function. The usefulness of this function is that it allows us to test for different levels of convexity with different values of $Q_{10}$; i.e., as $Q_{10}$ gets higher so does the second derivative of the function $R^{\prime \prime}$. We ran the model for $Q_{10}=2,3$, and 4 .

We also simulated respiration using the empirical functions implemented in the DAYCENT and CENTURY models (Del Grosso et al., 2005), given by

$$
R_{t}=F\left(T_{\text {soil }}\right) F(\text { RWC }) \text {, }
$$

where respiration $R_{t}$ is represented as the combined effect of soil relative water content (RWC) and temperature $\left(T_{\text {soil }}\right)$. These individual effects are represented as

$$
F\left(T_{\text {soil }}\right)=0.56+\left(1.46 \arctan \left(\pi 0.0309\left(T_{\text {soil }}-15.7\right)\right)\right) / \pi,
$$

$F(\mathrm{RWC})=5(0.287+(\arctan (\pi 0.009(\mathrm{RWC}-17.47))) / \pi)$.
Since we are only interested in exploring the effects of temperature variability, a constant water content of $\mathrm{RWC}=50 \%$ was assumed, so $F(\mathrm{RWC})=2.62$ in all simulations.

The benefit of using Eq. (6) is that it has an inflection point at $T=15.7$, which means the relationship between respiration and temperature changes from convex to concave at this value. The implication for the three ecosystems being modeled is that the range of temperatures for the arctic tundra is below this inflection value whereas the range for the tropical forest is above. For the temperate forest site, this inflection point lies in the middle of its temperature range.

Equations (4) and (5) were used to calculate total cumulative respiration for the whole simulation period $(10 \mathrm{yr})$ and compare differences between the reference $(\mathrm{A})$ and the new (B) climatic regime with the index

$$
\delta R=\frac{\sum_{t=1}^{3650} R_{t}^{\mathrm{B}}-\sum_{t=1}^{3650} R_{t}^{A}}{\sum_{t=1}^{3650} R_{t}^{\mathrm{A}}} .
$$


Results from all 600 simulations comparing the reference regime and a new climatic regime from all possible combinations of $m$ and $s$ are presented in a single MSC plot.

\section{Results}

\subsection{Analytical analysis}

\subsubsection{Geometric argument}

A real-valued function $f(x)$ is said to be convex on an interval $I$ if

$f[\lambda x+(1-\lambda) y] \leq \lambda f(x)+(1-\lambda) f(y)$,

for all $x, y \in I$, and $\lambda$ in the open interval $(0,1)$. Consider now the closed interval $[a, b]$ which is contained in the interval $[c, d] \in I$; both intervals with an average value $\bar{x}$, $c=a-h, d=b+h$, and

$\bar{x}=\lambda a+(1-\lambda) b=\lambda c+(1-\lambda) d$.

Using the definition of convexity in Eq. (9) we can show that

$f(\bar{x}) \leq \lambda f(a)+(1-\lambda) f(b) \leq \lambda f(c)+(1-\lambda) f(d)$.

This inequality can be confirmed graphically (Fig. 2) and analytically (Appendix). Geometrically, this inequality implies that the end points of two intervals with the same mean produce different means after convex transformation.

A change in variability of a random variable such as temperature implies a change in the interval of possible values that this variable can take. Equation (11) suggests that changes in variance alone, without changes in the average value of a random variable, produce different values of the average of all the function evaluations. Although this geometric argument is informative, the implications can be better studied in a probabilistic setting.

\subsubsection{Probabilistic argument}

Assume that respiration is a function of temperature $R=$ $f(T)$, which is a strictly convex function on the interval $I=(-\infty,+\infty)$, so by definition $f^{\prime \prime}(T)>0, \forall T \in I$. Let's now assume two random variables $T_{1}$ and $T_{2}$ that are normally distributed with equal mean but with different variance, so $T_{1} \sim N\left(\mu, \sigma_{1}^{2}\right)$ and $T_{2} \sim N\left(\mu, \sigma_{2}^{2}\right)$. Let's also assume that $\sigma_{1}^{2}>\sigma_{2}^{2}$. For simplicity, $T_{1}$ and $T_{2}$ can be transformed to $z_{1}=\left(T_{1}-\mu\right) / \sigma_{1}$ and $z_{2}=\left(T_{2}-\mu\right) / \sigma_{2}$, respectively. The expected value of $E\left[f\left(T_{1}\right)\right]=E\left[f\left(\sigma_{1} z_{1}\right)\right]$, and $E\left[f\left(T_{2}\right)\right]=E\left[f\left(\sigma_{2} z_{2}\right)\right]$ can be calculated as

$$
\begin{aligned}
& E\left[f\left(\sigma_{1} z_{1}\right)\right]=\int_{-\infty}^{\infty} f\left(\sigma_{1} z_{1}\right) \frac{1}{\sqrt{2 \pi}} \exp \left(\frac{-z_{1}^{2}}{2}\right) d z \\
& =\frac{1}{\sqrt{2 \pi}} \int_{0}^{\infty} f\left(\sigma_{1} z_{1}\right) \exp \left(\frac{-z_{1}^{2}}{2}\right) d z
\end{aligned}
$$

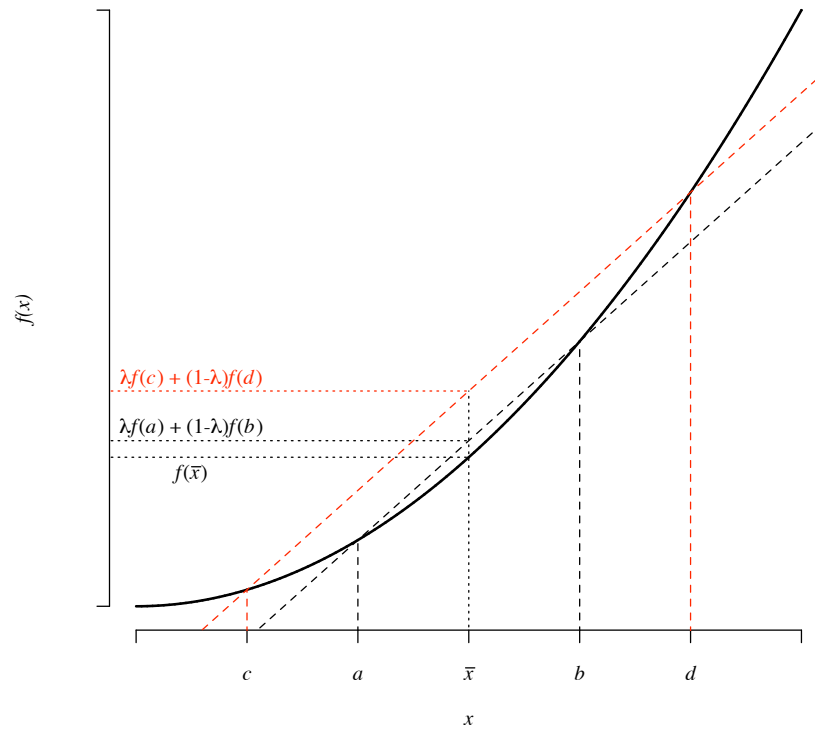

Fig. 2. Graphical representation of the inequalities in Eq. (11). $\bar{x}$ is the center point of both intervals $[a, b]$ and $[c, d]$.

$$
\begin{aligned}
& +\frac{1}{\sqrt{2 \pi}} \int_{-\infty}^{0} f\left(\sigma_{1} z_{1}\right) \exp \left(\frac{-z_{1}^{2}}{2}\right) d z, \\
& =\frac{1}{\sqrt{2 \pi}}\left[\int_{0}^{\infty}\left[f\left(\sigma_{1} z_{1}\right)+f\left(-\sigma_{1} z_{1}\right)\right] \exp \left(\frac{-z_{1}^{2}}{2}\right) d z\right] .
\end{aligned}
$$

Similarly,

$$
E\left[f\left(\sigma_{2} z_{2}\right)\right]=\frac{1}{\sqrt{2 \pi}}\left[\int_{0}^{\infty}\left[f\left(\sigma_{2} z_{2}\right)+f\left(-\sigma_{2} z_{2}\right)\right] \exp \left(\frac{-z_{2}^{2}}{2}\right) d z\right] .
$$

According to the properties of convexity it can be shown that $f\left(\sigma_{1} z_{1}\right)+f\left(-\sigma_{1} z_{1}\right)>f\left(\sigma_{2} z_{2}\right)+f\left(-\sigma_{2} z_{2}\right)$ (equation $11)$; therefore,

$E\left[f\left(T_{1}\right)\right]>E\left[f\left(T_{2}\right)\right]$.

Notice that the magnitude of the difference $\mid\left[f\left(\sigma_{1} z_{1}\right)+\right.$ $\left.f\left(-\sigma_{1} z_{1}\right)\right]-\left[f\left(\sigma_{2} z_{2}\right)+f\left(-\sigma_{2} z_{2}\right)\right] \mid$ depends on the degree of convexity of $f(T)$.

Alternatively, we can calculate the difference

$$
\begin{aligned}
\Delta & =E\left[f\left(T_{1}\right)\right]-E\left[f\left(T_{2}\right)\right] \\
& =\frac{1}{\sqrt{2 \pi}} \int_{0}^{\infty}\left\{\left[f\left(\mu+\sigma_{1} z\right)-f\left(\mu+\sigma_{2} z\right)\right]\right. \\
& \left.+\left[f\left(\mu-\sigma_{1} z\right)-f\left(\mu-\sigma_{2} z\right)\right]\right\} \exp \left(-\frac{z^{2}}{2}\right) d z, \\
& =\frac{1}{\sqrt{2 \pi}} \int_{0}^{\infty}\left(\sigma_{1}-\sigma_{2}\right) z\left[f^{\prime}(b(z))\right. \\
& \left.-f^{\prime}(a(z))\right] \exp \left(-\frac{z^{2}}{2}\right) d z,
\end{aligned}
$$


where $f^{\prime}(b(z))$ and $f^{\prime}(a(z))$ are derivatives of some points $a(z)$ and $b(z)$ for each $z>0$, such that

$$
\begin{aligned}
& \frac{f\left(\mu+\sigma_{1} z\right)-f\left(\mu+\sigma_{2} z\right)}{\left(\sigma_{1}-\sigma_{2}\right) z}=f^{\prime}(b(z)) \\
& \frac{f\left(\mu-\sigma_{1} z\right)-f\left(\mu-\sigma_{2} z\right)}{\left(\sigma_{1}-\sigma_{2}\right) z}=(-1) f^{\prime}(a(z)) .
\end{aligned}
$$

This is a consequence of the mean value theorem, so $\mu-\sigma_{1} z \leq a(z) \leq \mu-\sigma_{2} z$ and $\mu+\sigma_{2} z \leq b(z) \leq \mu+\sigma_{1} z$. It is important to note the -1 in front of the derivative at $a(z)$. Note also that since $a(z) \leq b(z)$, by the assumption of strict convexity $f^{\prime}(b(z))>f^{\prime}(a(z))$, or equivalently $f^{\prime}(b(z))-f^{\prime}(a(z))>0$; which in Eq. (15) results in $E\left[f\left(T_{1}\right)\right]-E\left[f\left(T_{2}\right)\right]>0$.

This analysis, summarized by Eq. (14), confirms the hypothesis initially posed: changes in temperature variance alone can produce differences in respiration depending on the degree of convexity between temperature and respiration.

The magnitude and functional relation of the effect of different variances can be evaluated by calculating the difference $\Delta=E\left[f\left(T_{1}\right)\right]-E\left[f\left(T_{2}\right)\right]$ for a particular respiration function. For simplicity, we will calculate $\Delta$ for the specific case of the exponential function $R=\exp (A T)$, where $A$ represents the degree of convexity of the function. Assuming $\sigma_{1}^{2}$ is $\eta$ times $\sigma_{2}^{2}\left(\sigma_{2}^{2}=\sigma_{1}^{2} / \eta\right)$

$$
\begin{aligned}
\Delta= & E\left[f\left(A \sigma_{1} z_{1}\right)\right]-E\left[f\left(A \sigma_{2} z_{2}\right)\right], \\
& =\frac{1}{\sqrt{2 \pi}} \int_{-\infty}^{\infty} f\left(A \sigma_{1} z_{1}\right) \exp \left(\frac{-z_{1}^{2}}{2}\right) d z \\
& -\frac{1}{\sqrt{2 \pi}} \int_{-\infty}^{\infty} f\left(A \sigma_{2} z_{2}\right) \exp \left(\frac{-z_{2}^{2}}{2}\right) d z, \\
& =\frac{1}{\sqrt{2 \pi}}\left[\sqrt{2 \pi} \exp \left(-A \sigma_{1}\right)-\sqrt{2 \pi} \exp \left(-A \sigma_{2}\right)\right], \\
& =\exp \left(-A \sigma_{1}\right)-\exp \left(-A \sigma_{1} / \eta\right) .
\end{aligned}
$$

Equation (17) summarizes the effects of a change in the variance of $T$ over $R$. It shows that both the degree of convexity $(A)$ and the magnitude of the change in variance $(\eta)$ affect the magnitude of the difference in expected values of respiration. As the degree of convexity increases, i.e. as $A$ gets bigger, the differences in respiration $(\Delta)$ are larger. In addition, an increase in variance $(\eta<1)$ produces an increase in the average respiration $(\Delta>0)$, and a decrease $(\Delta<0)$ when variance diminishes $(\eta>1)$ (Fig. 3).

Notice that the analysis above only holds for functions that are convex (or concave) in $I$. When the convexity of the function changes in the interval, different results can be obtained. The numerical example below helps to illustrate the effect of changes in convexity within $I$.

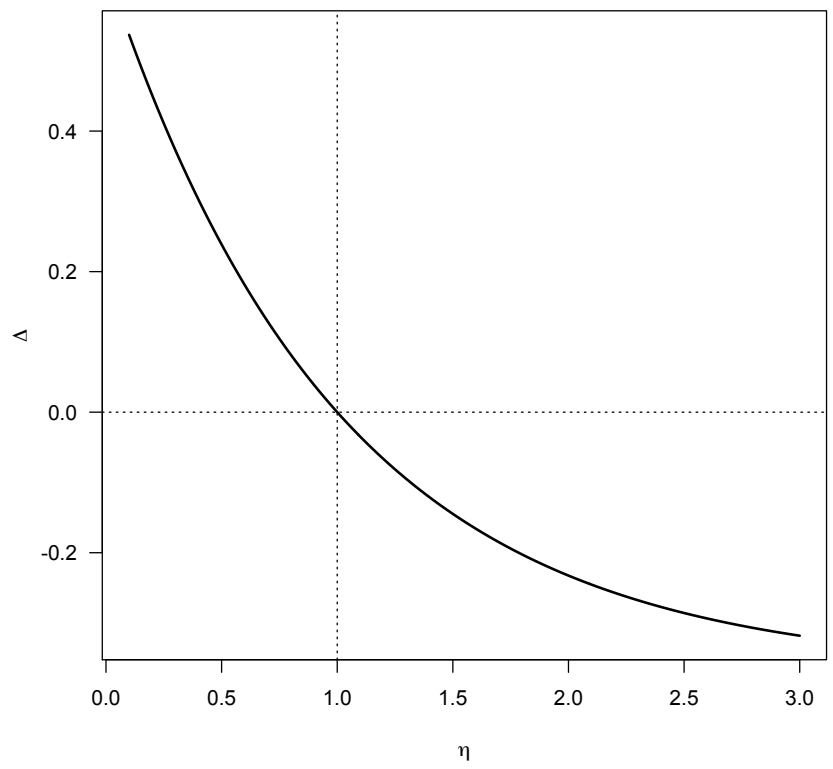

Fig. 3. Relationship between $\eta$ (proportional change in temperature variance) and $\Delta$ (difference in average respiration) for an exponential function $R=\exp (T)$.

\subsection{Numerical analysis}

Simulations were performed using two different respiration models. We used the modified van't Hoff model, which is a convex function for all the temperature values where biological processes take place (Table 1). We also used the empirical model implemented in the CENTURY and DAYCENT models (Del Grosso et al., 2005), which is a s-shaped relationship between temperature and soil respiration with a change in convexity at a value of $15.7^{\circ} \mathrm{C}$. The data for the arctic tundra lies in the convex range of the DAYCENT/CENTURY model, while the data for the temperate forest lies in the middle of the range where the change in convexity occurs, and the data for the tropical forests lies in the concave part of the range.

The results obtained using the modified van't Hoff model were similar for the three ecosystems modeled, so we will focus on the results for the temperate forest only. The differences in cumulative respiration between a reference climate and the set of new climatic regimes $(\delta R)$ confirm our initial expectation. First, the results from the simulations show that changes in the variance of temperature alone can increase the amount of carbon respired. This can be observed in Fig. 4 by changes of $\delta R$ as the standard deviation of temperature changes relative to the reference regime ( $S / S 0$, vertical axis). For example, an increase of about $6 \%$ of carbon respired over $10 \mathrm{yr}$ can be achieved by a 2.5 times increase in the standard deviation. Second, our simulations show that respiration is more sensitive to proportional changes in the average temperature $((T-T 0) / S 0$, vertical axis) than to changes in variance. The same $6 \%$ increase in respiration obtained 


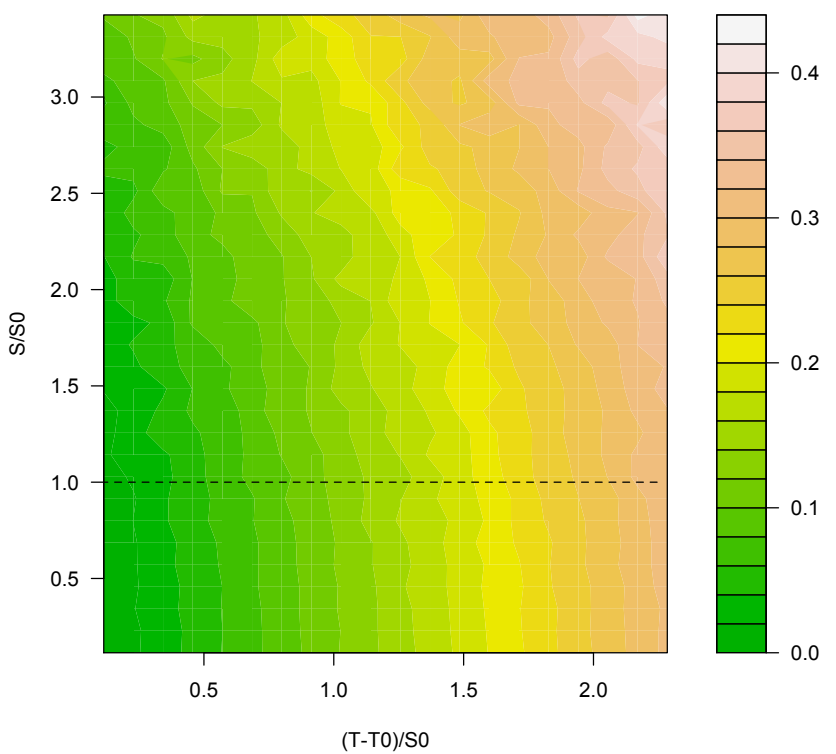

Fig. 4. Mean and Standard deviation Change (MSC) plot for H. J. Andrews using the modified van't Hoff equation with $Q_{10}=4$ showing standardized changes in the mean $(T-T 0) / S 0$ against standardized standard deviation $S / S 0$. Colors represent values of $\delta R$, i.e., the proportional increase in respiration over $10 \mathrm{yr}$ from one climatic regime over the other. The dashed horizontal line represents simulations in which variance remained constant relative to the reference climatic regime. $S / S 0>1$ represents increase in variance and $(T-T 0) / S 0>0$ represents increase in average temperature.

with a 2.5 times increase in standard deviation could have been achieved by increasing the average temperature by a factor of 0.5. Third, simultaneous changes of the average and the variance of temperature show that increases in variance amplify the effects of increases in the average. In contrast, decreases in variance dampen the effects of the increase in the average temperature. This can be observed in Fig. 4 by changes in $\delta R$ along the vertical direction at any fixed point in the horizontal axis.

The degree to which $\delta R$ responds to changes in variance was highly dependent on $Q_{10}$ (Fig. 5). As the value of $Q_{10}$ decreases the convexity of the function decreases (value of the second derivative gets smaller) and the effect of variability on respiration becomes less important. This behavior was consistent for the three ecosystems studied.

The approach used in the DAYCENT and CENTURY models produced different results for the three ecosystems analyzed. For the arctic tundra site the pattern observed was similar to the overall patterns observed with the van't Hoff equation (Fig. 6a). For the temperate forest though, the DAYCENT approach shows no sensitivity of respiration to temperature variance (Fig. 6b). For the tropical forest the results are the opposite, showing a dampening effect with increases in the average temperature and amplification with decreases in the average (Fig. 6c).
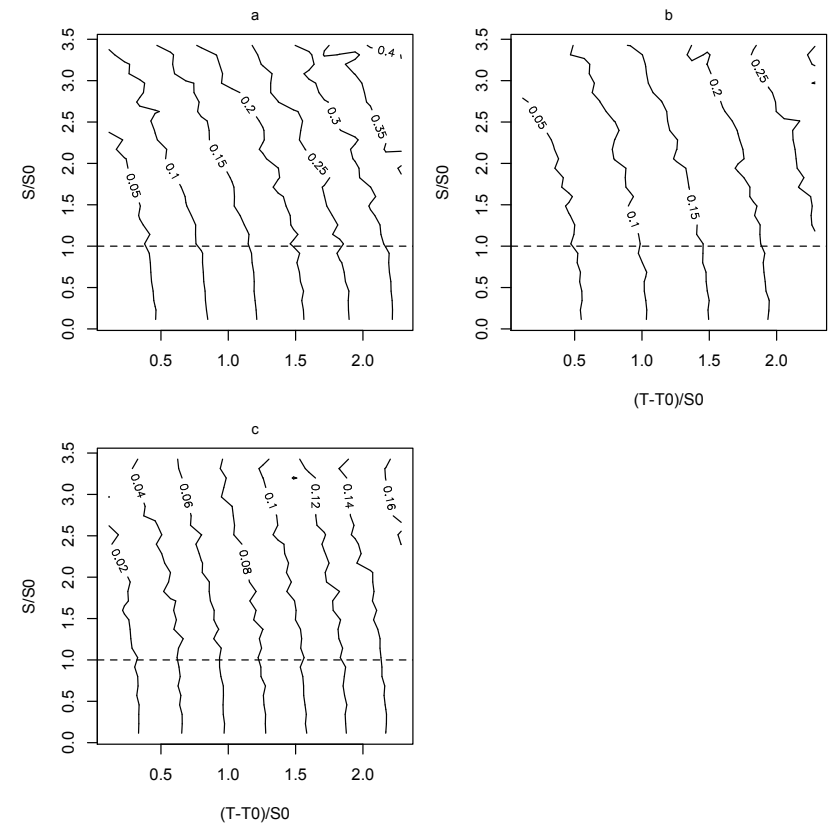

Fig. 5. MSC plots for H. J. Andrews using the modified van't Hoff equation and $Q_{10}=4,3,2$, for panels (a), (b), and (c), respectively. Contours represent values of $\delta R$, i.e., the proportional increase in respiration over $10 \mathrm{yr}$ from one climatic regime over the other.
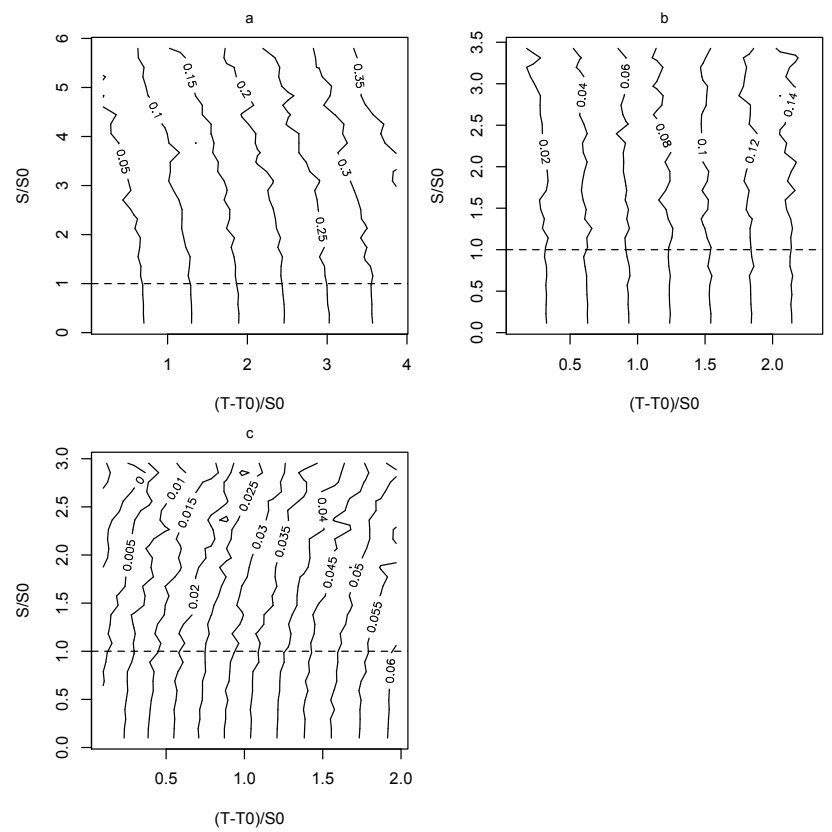

Fig. 6. MSC plots for Toolik lake (a), H. J. Andrews (b), and La Selva (c) using the DAYCENT and CENTURY approach. Contours represent values of $\delta R$, i.e., the proportional increase in respiration over $10 \mathrm{yr}$ from one climatic regime over the other. 


\section{Discussion}

The results from this analysis confirmed our initial expectations about possible effects of changes in temperature variance on respiration. First, we found that changes in the variance of temperature alone can either decrease or increase the amount of soil respiration in terrestrial ecosystems. The magnitude of this effect depends on both the degree of convexity or concavity between temperature and respiration (value of the second derivative of the relationship) and the magnitude of the change in variance. This was confirmed by both the analytical and numerical analyses. Second, changes in temperature variance can either amplify or dampen the effects of changes in the average temperature on soil respiration, again depending on the degree of convexity of the function and the magnitude of the change in variance.

\subsection{Climate variability and change}

Changes in climate variability and its effects on ecosystems have been studied less intensively than changes in the mean climate. The ongoing modification of the climatic system by changes in albedo, aerosols and prominently greenhouse gases, affects not only the mean state of the climate system but also its variability (Räisänen, 2002; Brönnimann et al., 2007; IPCC, 2007). Changes in temperature variability may result in changes in the frequency of events apart from the mean state such as unusual warm or cold events. Significant attention has been given to extreme climatic events such as heat waves or floods for their dramatic effects on populations. However, less extreme but still unusual events can have important implications for the functioning of ecosystems over the long-term (Jentsch et al., 2007).

Important changes have been observed in temperature variability for different regions in the recent past. In Europe, where climate records are longer than in other continents, important changes in the variance of temperature have been described. Della-Marta and Beniston (2007) report an increase in the variance of summer maximum temperature of $6^{\circ} \mathrm{C}$ for the period 1880-2005 in Western Europe. This increase in variance accounts for at least $40 \%$ of the increase in the frequency of hot days after accounting for the increase in the average temperature. In Central Europe there is evidence of current changes in temperature variance (Schar et al., 2004), and most climate models predict increases in summer temperature variability for the 21 st century (Scherrer et al., 2007). Increasing trends in temperature variability are also emerging in temperature time-series from North America (Meehl et al., 2009).

Changes in climate variability are not expected to be homogenous globally. A comparison of 19 atmosphereocean general circulation models (AOGCMs) showed that as a consequence of doubling $\mathrm{CO}_{2}$, temperature variability is expected to decrease during the winter of the ex- tratropical Northern Hemisphere (Räisänen, 2002). Conversely, the AOGCMs show that temperature variability is expected to increase during the summer in low latitudes and northern midlatitudes. A more recent analysis using an updated version of one climate model confirms these results, which also suggest decreases in temperature variability for fall, spring and winter in the Northern Hemisphere (Stouffer and Wetherald, 2007).

These expected changes in temperature variability most likely will have consequences on the total amount of carbon emitted to the atmosphere from terrestrial ecosystems. Our results showed that changes in variance produce a variety of effects on respiration in addition to the predicted effects of changes in average temperatures alone. At a global scale, differences in temperature sensitivities of respiration and in the magnitude of change in temperature variance for different regions will produce a variety of responses. For example, at high latitudes in the Northern Hemisphere, where the mean temperature is expected to increase and the variance is expected to decrease, respiration would probably be lower than the predicted by changes in mean temperature alone. These regions are expected to contribute the most to the positive feedback between temperature and respiration, but this effect may be overestimated in previously published analyses. At lower latitudes, where both the mean and variance of temperature are expected to increase, the amount of respiration would be higher than the predicted by changes in mean temperature alone. These predictions obviously depend on the assumption of a convex relationship between respiration and temperature.

\subsection{Convexity of respiration functions}

The relationship between temperature and respiration historically has been described using empirical convex functions (Table 1). Although there have been important criticisms to simple empirical models and more mechanistic representations are currently being discussed (e.g., Luo and Zhou, 2006; Davidson et al., 2006), it is clear from the many modeling approaches that these two variables have a convex relationship, independent of the type of model employed. In some cases, however, this relationship shows a change to concavity at higher values of temperature, which could be explained by the interacting effects of soil water content and substrate availability (Del Grosso et al., 2005; Davidson et al., 2006), among other factors.

Independent of the type of model being used, the number of pools represented, or the main drivers included in the model, the results obtained in this study will apply to all predictions of future respiration if its relationship with temperature is other than a simple straight line. They also apply to all temperature ranges where most biological process take place, except where the respiration function changes from convex to concave. As the simulations in the temperate forest showed, 
a change in convexity can cause the effects of concavity at one part of the temperature range to compensate the effects of convexity in the other part of the range.

In general, simulation models use one single equation to described the relationship between temperature and respiration, with the modified van't Hoff model being the most common. Although there is important work trying to predict $Q_{10}$ values for different ecosystem types (e.g., Chen and Tian, 2005; Fierer et al., 2006), this modeling approach only considers one single functional relationship between temperature and respiration. For some systems it is possible that this relationship may be either other than convex or change to concavity at some point in the temperature range; therefore, more effort should be directed to understand how local scale relationships can be incorporated in models and whether these relationships are generalizable within and across major biomes.

\section{Implications}

Although ecologists are well aware of the importance of climate variability (e.g. Rastetter et al., 1992; Kicklighter et al., 1994; Ruel and Ayres, 1999; Pasztor et al., 2000; Knapp et al., 2002; Jentsch et al., 2007), studies looking at the effects of climate change on ecosystem function have given perhaps too much attention to changes in the average climate, but not to the full probability distribution of the climate system. The findings of this study can greatly modify past predictions about the effects of future average temperatures on ecosystem respiration, especially for large temporal and spatial scales. However, soil respiration not only depends on temperature but also on moisture and substrate availability. New climatic regimes will be associated with different soil moisture regimes and different plant phenologies that control substrate supply. There has been a considerable amount of work showing the effects of variable soil moisture on respiration through drying/wetting cycles (see Borken and Matzner, 2009 for a review). If combined with the results from this study, changes in both temperature and precipitation variance would likely produce complex behaviors not incorporated in current model predictions. In coupled atmosphere-biosphere models though, the implications may be less drastic since the time-steps in these models are generally short enough to account for climatic variability and consequently reduce bias.

The effects of new climatic regimes with different variances would not only affect the mineralization of $\mathrm{C}$ measured as soil respiration, but also the production of dissolved organic and inorganic forms of $\mathrm{C}$ as well as other elements such as N. Autotrophic respiration is also likely to be affected by changes in the variance of temperature since they usually correlate well with a modified van't Hoff model (Ryan, 1991). In general, any ecosystem process that is related with a climatic variable through a convex or concave function is likely to be subject to the effects of changes in variance presented here.

In terms of whole ecosystem carbon balance, changes in climatic regimes can also affect the rates of carbon uptake. The effect of changes in the variance of different climatic variables on photosynthesis can offset the changes produced on respiration (Medvigy et al., 2010). For analyses at the ecosystem level, the analytical framework provided here can serve as a basis to identify the functions that would present a larger response to the variance of climatic variables by studying the degree of convexity of these functions and the expected changes in variance of those variables.

For better predictions of future soil respiration based on possible changes in temperature variance it is recommended that models are run with high-frequency data, which does not require modifications in model structure, but instead in the type of data used in the simulations. High-frequency data with explicit changes in variance can be obtained in different ways: (1) for predictions of past respiration fluxes at a site, data from meteorological stations, eddy-flux towers, or automated systems for soil monitoring can be used to predict high-frequency respiration fluxes. The temperature data can be plotted in a MSC diagram, as in our study, to explore possible changes in temperature variance. (2) For predictions of past respiration fluxes at larger scales, re-analysis highfrequency data can be used. These data can be obtained in 6hourly format from the National Center for Atmospheric Research's website or from the European Centre for MediumRange Weather Forecasts. Again, the MSC plots can be used with these data to explore past changes in temperature variance. (3) A random weather generator can be used for exploring future changes in the variance of temperature. The procedure is basically the same as the used in the numerical simulations in our analysis. It consists of sampling random numbers from a probability distribution with known mean and variance. The values of variance can be changed over time to simulate plausible changes in the climatic regime.

\section{Conclusions}

In this study we found simple and general mathematical expressions to predict the effects of changes in temperature variance on the amount of carbon respired from soils.

Assume two temperature regimes $T_{1}$ and $T_{2}$, both with the same mean value, but with different variances, so $\operatorname{Var}\left(T_{1}\right)<\operatorname{Var}\left(T_{2}\right)$. A general statement about average respiration rates, which is an extension of Jensen's inequality derived from this analysis, is that

$E\left(f\left(T_{1}\right)\right)<E\left(f\left(T_{2}\right)\right)$.

This inequality implies that increases in temperature variance without changes in the average temperature result in an increase of the average respiration, provided that the relationship between temperature and respiration is a convex 
function. For concave functions the sign of the inequality is reversed, which results in a decrease in average respiration with increases in temperature variance.

Another generalization, which can be deduced from the numerical analysis, is that changes in temperature variance amplify or dampen the effects of changes in average temperature. If the average temperature increase in proportion to a quantity $\eta>1$, then

$\eta E\left(f\left(T_{1}\right)\right)<E\left(f\left(\eta T_{1}\right)\right)<E\left(f\left(\eta T_{2}\right)\right)$.

The left hand side inequality is a special case of the nonhomogeneity property of nonlinear systems. In the context of this study, it implies that the average respiration caused by a proportional increase in the average temperature is higher that the same proportional increase of respiration under average temperature conditions. The right-hand side of Eq. (19) implies that the average respiration is higher if the variance of temperature increases. The sign of the inequality is reversed if the system is concave or the variance of $T_{1}$ is lower than the variance of $T_{2}$.

From this set of inequalities it is possible to predict under what circumstances changes in temperature variance cause amplification or dampening of average respiration rates.

\section{Appendix A}

\section{Derivation of geometric inequality}

The point $\bar{x}$ can be defined as the average value of both $[a, b]$ and $[c, d]$, so $\bar{x}=\lambda a+(1-\lambda) b=\lambda c+(1-\lambda) d$. Applying the definition of convexity we can see that

$f(\bar{x})=f(\lambda a+(1-\lambda) b) \leq \lambda f(a)+(1-\lambda) f(b)$.

Now, the points $a$ and $b$ can be expressed as a linear combination of $c$ and $d$

$a=\alpha c+(1-\alpha) d$,

$b=(1-\beta) c+\beta d$.

Given that $\alpha=\beta$, and $\lambda+(1-\lambda)=1 ; \lambda$ and $(1-\lambda)$ can be expressed in terms of $\alpha$ and $\beta$ since the following expressions apply

$\alpha+(1-\alpha)=1$,

$\alpha+(1-\beta)=1$,

$[\lambda+(1-\lambda)][\alpha+(1-\beta)]=1$,

$[\lambda \alpha+(1-\lambda)(1-\beta)]+[\lambda(1-\beta)+\alpha(1-\lambda)]=1$,

and again, since $\lambda+(1-\lambda)=1$ we obtain

$$
\begin{aligned}
\lambda & =\lambda \alpha+(1-\lambda)(1-\beta), \\
(1-\lambda) & =\lambda(1-\beta)+\alpha(1-\lambda) .
\end{aligned}
$$

We can now express the right-hand side of Eq. (A1) in terms of $c$ and $d$ as

$$
\begin{aligned}
\lambda f(\alpha c+ & (1-\alpha) d)+(1-\lambda) f((1-\beta) c+\beta d) \\
& \leq \lambda \alpha f(c)+\lambda(1-\alpha) f(d) \\
& +(1-\lambda)(1-\beta) f(c)+(1-\lambda) \beta f(d), \\
& \leq f(c)[\lambda \alpha+(1-\lambda)(1-\beta)] \\
& +f(d)[\lambda(1-\alpha)+(1-\lambda) \beta], \\
& \leq \lambda f(c)+(1-\lambda) f(d) .
\end{aligned}
$$

Therefore,

$f(\bar{x}) \leq \lambda f(a)+(1-\lambda) f(b) \leq \lambda f(c)+(1-\lambda) f(d)$.

Acknowledgements. Financial support was provided by the Eduardo Ruiz Landa Fellowship and the Kay and Ward Richardson endowment. Data used in this research was also supported by funding from the NSF Long-term Ecological Research (LTER) Program to the H. J. Andrews and Toolik Lake LTER sites. We would like to thank Claire Phillips and Michael Unsworth for important comments on previous versions of this manuscript.

The service charges for this open access publication have been covered by the Max Planck Society.

Edited by: P. Stoy

\section{References}

Ågren, G. I. and Axelsson, B.: Population respiration: A theoretical approach, Ecol. Model., 11, 39-54, 1980.

Borken, W. and Matzner, E.: Reappraisal of drying and wetting effects on $\mathrm{C}$ and $\mathrm{N}$ mineralization and fluxes in soils, Glob. Change Biol., 15, 808-824, doi:10.1111/j.1365-2486.2008.01681.x, 2009.

Brönnimann, S., Ewen, T., Luterbacher, J., Diaz, H. F., Stolarski, R. S., and Neu, U.: A Focus on Climate During the Past 100 Years, in: Climate Variability and Extremes during the Past 100 Years, edited by: Brönnimann, S., Luterbacher, J., Ewen, T., Diaz, H. F., Stolarski, R. S., and Neu, U., Springer, 1-25, doi:10.1007/978-1-4020-6766-2_1, 2007.

Chen, H. and Tian, H.-Q.: Does a General Temperature-Dependent Q10 Model of Soil Respiration Exist at Biome and Global Scale?, J. Integr. Plant Biol., 47, 1288-1302, 2005.

Davidson, E. A. and Janssens, I. A.: Temperature sensitivity of soil carbon decomposition and feedbacks to climate change, Nature, 440, 165-173, doi:10.1038/nature04514, 2006.

Davidson, E. A., Janssens, I. A., and Luo, Y.: On the variability of respiration in terrestrial ecosystems: moving beyond Q10, Glob. Change Biol., 12, 154-164, doi:10.1111/j.13652486.2005.01065.x, 2006.

Del Grosso, S. J., Parton, W. J., Mosier, A. R., Holland, E. A., Pendall, E., Schimel, D. S., and Ojima, D. S.: Modeling soil $\mathrm{CO}_{2}$ emissions from ecosystems, Biogeochemistry, 73, 71-91, doi:10.1007/s10533-004-0898-z, 2005.

Della-Marta, P. M. and Beniston, M.: Summer Heat Waves in Western Europe, Their Past Change and Future Projections, in: Climate Variability and Extremes during the Past 100 Years, edited 
by: Bronnimann, S., Luterbacher, J., Ewen, T., Diaz, H. F., Stolarski, R. S., and Neu, U., Springer, 235-250, doi:10.1007/9781-4020-6766-2_16, 2007.

Field, C. B., Lobell, D. B., Peters, H. A., and Chiariello, N. R.: Feedbacks of Terrestrial Ecosystems to Climate Change*, Annu. Rev. Env. Resour., 32, 1-29, 2007.

Fierer, N., Colman, B. P., Schimel, J. P., and Jackson, R. B.: Predicting the temperature dependence of microbial respiration in soil: A continental-scale analysis, Glob. Biogeochem. Cy., 20, GB3026, doi:10.1029/2005GB002644, 2006.

Houghton, R. A.: Balancing the Global Carbon Budget, Annu. Rev. Earth Pl. Sc., 35, 313-347, 2007.

IPCC: Climate Change 2007: The Physical Science Basis. Contribution of Working Group I to the Fourth Assessment Report of the Intergovernmental Panel on Climate Change, Cambridge University Press, Cambridge, United Kingdom and New York, NY, 2007.

Jensen, J.: Sur les fonctions convexes et les inégalités entre les valeurs moyennes, Acta Mathematica, 30, 175-193, doi:10.1007/BF02418571, 1906.

Jentsch, A., Kreyling, J., and Beierkuhnlein, C.: A new generation of climate-change experiments: events, not trends, Front. Ecol. Environ., 5, 365-374, 2007.

Kicklighter, D. W., Melillo, J. M., Peterjohn, W. T., Rastetter, E. B., McGuire, A. D., Steudler, P. A., and Aber, J. D.: Aspects of spatial and temporal aggregation in estimating regional carbon dioxide fluxes from temperate forest soils, J. Geophys. Res., 99, 1303-1315, doi:10.1029/93JD02964, 1994.

Kirschbaum, M. U. F.: The temperature dependence of soil organic matter decomposition, and the effect of global warming on soil organic C storage, Soil Biol. Biochem., 27, 753-760, doi:10.1016/0038-0717(94)00242-S, 1995.

Knapp, A. K., Fay, P. A., Blair, J. M., Collins, S. L., Smith, M. D., Carlisle, J. D., Harper, C. W., Danner, B. T., Lett, M. S., and McCarron, J. K.: Rainfall Variability, Carbon Cycling, and Plant Species Diversity in a Mesic Grassland, Science, 298, 22022205, 2002.

Kucera, C. L. and Kirkham, D. R.: Soil Respiration Studies in Tallgrass Prairie in Missouri, Ecology 52, 912-915, 1971.

Lloyd, J. and Taylor, J. A.: On the Temperature Dependence of Soil Respiration, Funct. Ecol., 8, 315-323, 1994.

Luo, Y. and Zhou, X.: Soil respiration and the environment, Academic Press, 2006.

Medvigy, D., Wofsy, S. C., Munger, J. W., and Moorcroft, P. R.: Responses of terrestrial ecosystems and carbon budgets to current and future environmental variability, P. Natl. Acad. Sci., 107, 8275-8280, 2010.

Meehl, G. A., Tebaldi, C., Walton, G., Easterling, D., and McDaniel, L.: Relative increase of record high maximum temperatures compared to record low minimum temperatures in the US, Geophys. Res. Lett., 36, L23701, doi:10.1029/2009GL040736, 2009.
Notaro, M.: Response of the mean global vegetation distribution to interannual climate variability, Clim. Dynam., 30, 845-854, doi:10.1007/s00382-007-0329-7, 2008.

Pasztor, L., Kisdi, E., and Meszena, G.: Jensen's inequality and optimal life history strategies in stochastic environments, Trends Ecol. Evol., 15, 117-118, doi:10.1016/S0169-5347(99)01801-7, 2000.

Räisänen, J.: $\mathrm{CO}_{2}$-Induced Changes in Interannual Temperature and Precipitation Variability in 19 CMIP2 Experiments, J. Climate, 15, 2395-2411, 2002.

Rastetter, E. B., King, A. W., Cosby, B. J., Hornberger, G. M., O'Neill, R. V., and Hobbie, J. E.: Aggregating Fine-Scale Ecological Knowledge to Model Coarser-Scale Attributes of Ecosystems, Ecol. Appl., 2, 55-70, doi:10.2307/1941889, 1992.

Ruel, J. J. and Ayres, M. P.: Jensen's inequality predicts effects of environmental variation, Trends Ecol. Evol., 14, 361-366, 1999.

Ryan, M. G.: Effects of Climate Change on Plant Respiration, Ecol. Appl., 1, 157-167, 1991.

Sardeshmukh, P. D., Compo, G. P., and Penland, C.: Changes of Probability Associated with El Niño, J. Climate, 13, 4268-4286, 2000.

Schar, C., Vidale, P. L., Luthi, D., Frei, C., Haberli, C., Liniger, M. A., and Appenzeller, C.: The role of increasing temperature variability in European summer heatwaves, Nature, 427, 332 336, doi:10.1038/nature02300, 2004.

Scherrer, S. C., Liniger, M. A., and Appenzeller, C.: Distribution Changes of Seasonal Mean Temperature in Observations and Climate Change Scenarios, in: Climate Variability and Extremes during the Past 100 Years, edited by: Brönnimann, S., Luterbacher, J., Ewen, T., Diaz, H. F., Stolarski, R. S., and Neu, U., Springer, 251-267, 2007.

Schimel, D. S., Braswell, B. H., Holland, E. A., McKeown, R., Ojima, D. S., Painter, T. H., Parton, W. J., and Townsend, A. R.: Climatic, Edaphic, and Biotic Controls Over Storage and Turnover of Carbon in Soils, Glob. Biogeochem. Cy., 8, 279293, doi:10.1029/94GB00993, 1994.

Sierra, C. A., Loescher, H. W., Harmon, M. E., Richardson, A. D., Hollinger, D. Y., and Perakis, S. S.: Interannual variation of carbon fluxes from three contrasting evergreen forests: the role of forest dynamics and climate, Ecology, 90, 2711-2723, 2009.

Stouffer, R. J. and Wetherald, R. T.: Changes of Variability in Response to Increasing Greenhouse Gases, Part I: Temperature, J. Climate, 20, 5455-5467, 2007.

Trumbore, S. E.: Potential responses of soil organic carbon to global environmental change, P. Natl. Acad. Sci. USA, 94, 8284-8291, 1997. 